\title{
Innovative electron microscope for light-element atom visualization
}

\section{— Development of low-voltage electron microscopes in Triple-C project -}

\author{
YUTA SATO", Takeo SASAKI ${ }^{2}$, Hidetaka SAWAdA ${ }^{2}$, Fumio HosokaWA ${ }^{2}$, Takeshi Tomita ${ }^{2}$, \\ Toshikatsu KANEYAMA ${ }^{2}$, Yukihito KONDO ${ }^{2}$ and Kazutomo SUENAGA ${ }^{1 *}$
}

[Translation from Synthesiology, Vol.4, No.3, p.166-175 (2011)]

\begin{abstract}
Today, the demand for techniques to directly visualize the atomic-level structures of nano-materials and so-called soft matter (organic molecules, bio-materials, etc.) is rapidly increasing. Observing these objects using conventional transmission electron microscopes (TEM) and scanning TEM (STEM) often results in serious irradiation-induced structural damage, and the images produced have an unsuitable contrast due to the high electron-acceleration voltages. We believe that reducing the acceleration voltages to several tens of kilovolts will enable direct imaging with less damage and help produce images with a higher contrast. However, correcting various aberrations, such as spherical and chromatic aberrations (Cs and Cc, respectively) and high-order geometrical astigmatisms, is necessary to achieve atomiclevel spatial resolution. In our Triple-C project, we have developed low-voltage TEM/STEM systems equipped with new Cs and Cc correctors for carbon-based nano-materials.
\end{abstract}

Keywords : Structure characterization, electron microscopy, aberration correction, nano-material, soft matter, single molecule, single atom, electronic state

\section{Introduction}

Currently, electron microscopy plays an increasingly important role in the characterization of nano- and bio-materials amidst the acceleration of research and development in these fields. High-performance transmission electron microscope (TEM) and scanning TEM (STEM) are expected to enable the direct visualization of structures at the single-atom/molecule level, particularly for carbon nano-materials (carbon nanotubes (CNTs), graphenes, etc.) and so-called soft matter (e.g., organic molecules and biorelated materials). The technology employed in electron microscopy ${ }^{[1][2]}$ has improved considerably since the 1970 s. However, previous technological developments were generally aimed at achieving higher spatial resolution by employing ultra-high electron acceleration voltages. The use of electron microscopes that were once considered cuttingedge and operated at ultra-high voltages in the order of a million volts (MV) has become limited to the observation of thick crystalline materials that are generally stable under electron-beam irradiation, such as metals, alloys, and inorganic compounds. On the other hand, the observation of non-crystalline light element materials such as soft matter by using ultra-high voltage microscopes has often been hindered by serious irradiation-induced damage to the specimens and insufficient signal intensity. The development of innovative electron microscopes is essential for overcoming these technical difficulties as well as for directly observing the dynamic behavior of single molecules and for detecting and identifying single light-element atoms.

We recognize the advantage of using low acceleration voltages for observing light-element materials such as carbon nano-materials and have pioneered techniques for directly imaging their atomic-level structures using TEMs and STEMs with $120 \mathrm{kV}$ operation. In 2004, single gadolinium (Ga) atoms incorporated into carbon nano-materials were identified by electron energy-loss spectroscopy (EELS) using a $\mathrm{STEM}^{[3]}$, and hexagonal networks of carbon atoms in CNTs were unambiguously detected by a $\mathrm{TEM}^{[4]}$. In 2007, individual carbon atoms that formed six-member rings in CNTs were visualized using a TEM equipped with a spherical aberration corrector ${ }^{[5][6]}$. In 2008, carbon hexagonal networks in CNTs were imaged using an aberration-corrected TEM operated at an even lower acceleration voltage of $80 \mathrm{kV}^{[7]}$. TEM images of single-walled CNTs (SWCNTs) observed under the conditions employed in these studies are shown in Fig. 1, where the effects of spherical aberration correction and different acceleration voltages on spatial resolution and image contrast are clearly found.

Currently available TEM and STEM systems have contributed to the characterization of nano-materials, as mentioned above. However, if we presume that they will be applied to the high-resolution imaging of soft matter such as bio-molecules, they need to be further developed

1. Nanotube Research Center, AIST Tsukuba Central 5, 1-1-1 Higashi, Tsukuba 305-8565, Japan

* E-mail : suenaga-kazu@aist.go.jp, 2. JEOL Ltd. 3-1-2 Musashino, Akishima 196-8558, Japan

Original manuscript received July 7, 2011, Revisions received August 22, 2011, Accepted August 23, 2011 
to decrease the irradiation-induced damage to specimens and improve both spatial and time resolution. No existing electron microscope is specially designed for characterizing such light-element materials. It is therefore essential to develop innovative microscopes based on concepts that are completely different from those used for previous ultra-high voltage microscopes, in order to realize the direct imaging and identification of individual atoms and molecules of soft matter. Based on these requirements, we attempt to overcome two difficult parameters that have been considered incompatible with each other with regard to conventional electron microscopes: extremely low electron acceleration voltage and atomic resolution. In 2006, we launched a full-scale project supported by JST-CREST ${ }^{[8]}$ to develop new electron microscopes suitable for the high-resolution observation of soft matter with less damage to the specimens and increased sensitivity. This is the first project aimed at constructing completely new electron microscopes that are specially designed to work at low acceleration voltages.

\section{Scenario for achieving our research goal}

Our project to develop new low-voltage electron microscopes is supported by JST-CREST ${ }^{[8]}$. It was started in October 2006 and will end in March 2012. The originally planned design for a low-voltage microscope and its presumed application are schematically shown in Fig. 2, in which Cs and Cc denote spherical and chromatic aberrations, respectively. Based on the currently available TEM/STEM systems and the problems associated with them, we have decided to intensively develop the following three components as the most important elemental technologies for the first stage of this project.

- Low acceleration voltage electron gun: highly stable at $30-60 \mathrm{kV}$ with excellent monochromaticity and luminance.

- A spherical aberration corrector having higher correction performance than existing products to fully compensate for the disadvantages affecting spatial resolution due to the use of low acceleration voltages.
- A chromatic aberration corrector having a new optical system to realize chromatic aberration correction that has been almost unprecedented so far $^{[9]}$.

During the second stage of the project, low-voltage electron microscopes were constructed by integrating the components including those picked up above, and their performance was examined. We originally planned to employ a TEM/STEM dual-use model equipped with both spherical and chromatic aberration correctors, as shown in Fig. 2. We later revised the plan to be more practicable and more efficient based on the progress made in the first stage, and we finally constructed two microscopes with different setups optimized for their respective applications. The performance of these microscopes was examined with the main consideration of how closely the spatial resolution $d$ approaches its critical value, as determined by the acceleration voltage $E$. Therefore, instead of $d$, we focused on the ratio $d / \lambda$, where $\lambda$ denotes the wavelength of electrons accelerated at $E$. Taking account that the highest spatial resolution of $d=0.05 \mathrm{~nm}$ previously recorded at $300 \mathrm{kV}^{[10]}$ corresponds to $d / \lambda=25$, we aimed to obtain a $d / \lambda$ smaller than 25 at a much lower $E$ by using our new microscope.

After the completion of the performance examination of the microscopes, this project has now entered the third stage, where the designed microscopes are practically used for the observation and analysis of various nano- and light-element materials. Here, we intend to demonstrate the advantage of our low-voltage microscopes by providing scientifically valuable results that can appeal to a wide audience. We primarily use carbon nano-materials as specimens to verify the effects of using low acceleration voltages in TEM, STEM, and EELS observations, while referring to our extensive data on the same materials previously obtained by using conventional microscopes with higher voltages. At the same time, we assign this stage for detecting and solving possible latent problems in our microscopes to make them more practicable. (a)

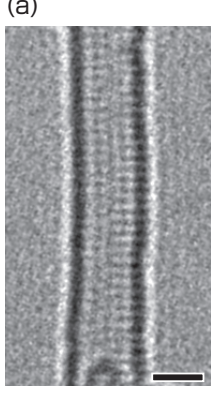

(b)

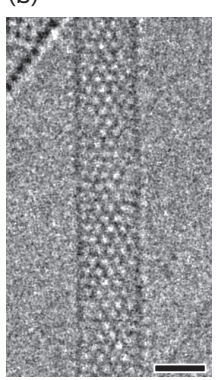

(c)

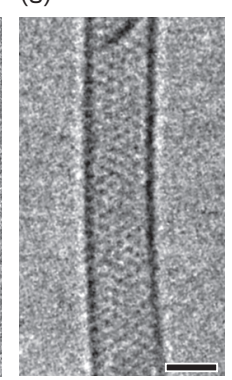

Fig. 1 Comparison of TEM images of SWCNTs (a) Recorded at an acceleration voltage of $120 \mathrm{kV}$ without spherical aberration (Cs) correction, (b) recorded at $120 \mathrm{kV}$ with Cs correction, (c) recorded at $80 \mathrm{kV}$ with Cs correction Scale bar $=1 \mathrm{~nm}$

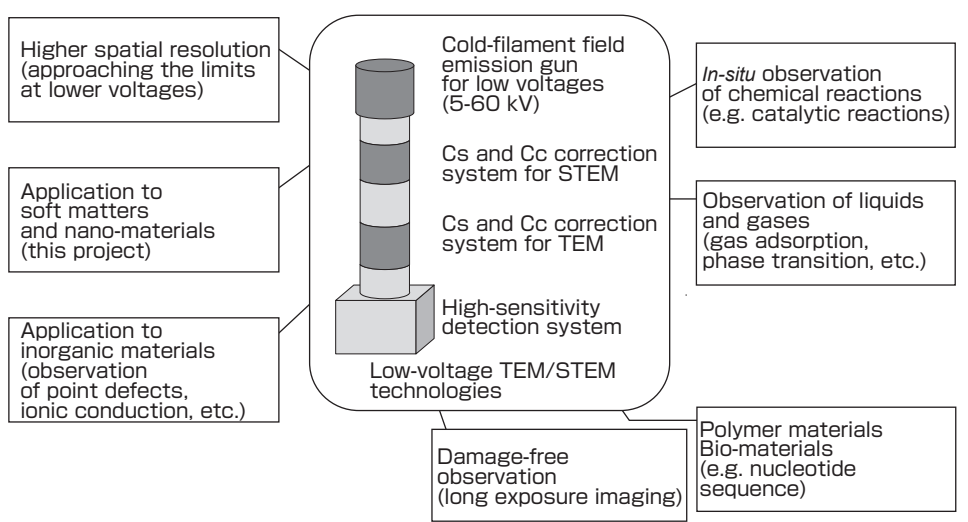

Fig. 2 Outline of low-voltage TEM/STEM and future prospects 
This project has been implemented, by following the scenario outlined above, to realize next-generation, high-performance low-voltage electron microscopes based on technologies that have all been developed within Japan. We named the project Triple-C because it focuses on the following three C's: spherical aberration (Cs), chromatic aberration (Cc), and carbon (C) nano-materials. This project has been carried out through a collaboration between JEOL Ltd., The National Institute for Material Science (NIMS), and the National Institute of Advanced Industrial Science and Technology (AIST). This project allows us to combine the expertise of all these organizations, such as the theories on electron microscopy and spectroscopy, experience in developing instruments, knowledge of nano-material science and experimental skills. The JEOL team developed each component during the first stage, and constructed the microscopes and examined their performances during the second stage. The NIMS and AIST teams took charge of the preliminary planning of this project, the application of the low-voltage microscopes to nano-materials during the third stage, and reference experiments using conventional microscopes. To achieve the best results from this joint project, we fully cooperated with each other for each experiment and shared information through meetings that were held approximately every two months to check on the project's progress.

In this paper, we review the development and performance of new low-voltage microscopes as carried out by the JEOL team as the principal part of our Triple-C project. We also introduce some typical scientific results and problems associated with the microscopes with regard to the characterization of carbon nano-materials by the AIST team.

\section{Elemental technologies}

\subsection{Low-voltage electron gun}

The electron gun of a microscope has the important function of stably generating and accelerating an electron beam at a fixed voltage. It is required, in particular, for the electron guns in the Triple-C project to produce a high luminance (current density) and a small energy spread $(\Delta E)$. High luminance is necessary for identifying light-element single atoms by STEM-EELS at a high signal-to-noise ratio $(\mathrm{S} / \mathrm{N})$. A small $\Delta E$ contributes to a minimization of the blurring of images due to the chromatic aberration of the lens system. Assuming that the acceleration voltage $E$ slightly changes to $E+d E$, the degree of electronbeam broadening due to chromatic aberration is proportional to $d E / E$. This implies that spatial resolution of a microscope is more seriously affected by chromatic aberration at a lower $E$. It is therefore essential to make the $\Delta E$ of the low-voltage electron gun as small as possible. Note that $\Delta E$ is generally evaluated as the full width at half maximum of the EELS zeroloss peak in electronvolts.

We adopted cold filament field-emission guns (CFEG) for our low-voltage microscopes because they have great advantages both in luminance and energy spread. The electron emitter and construction of the accelerating tubes, which apply voltages to electrons, were optimized for use at 30-60 kV. We also implemented measures for reducing the noise in hightension electric sources and circuits for higher CFEG stability. As a result, excellent energy spreads of 0.27 and $0.30 \mathrm{eV}$ were obtained at acceleration voltages of 60 and $30 \mathrm{kV}$, respectively, as shown in the EELS zero-loss peaks in Fig. $3^{[11]}$.

\subsection{Spherical aberration corrector}

In electron microscope lenses, magnetic fields are applied to focus the electron beam by using the refracting effect of Lorentz forces in the fields. Although the electron beam passing through the lens system should ideally converge on a certain point along the optical axis, the focal point is actually spread out to some extent by various aberrations of the lenses, resulting in blurring and/or distortion of the projected images. In particular, spherical aberration of the objective lens of TEM is a major limiting factor of the spatial resolution. Recently, the correction of spherical aberration has been realized by using several stages of magnetic multipoles, which were incorporated in the rear of the objective lens in the microscope column ${ }^{[12]-[14]}$. This correction system itself generates negative spherical aberration to cancel the original positive aberration of the lens. A commercially available spherical aberration corrector developed by CEOS $\mathrm{GmbH}$, which has become more popular both for TEM and STEM in recent years, consists of double hexapoles and transfer lenses. Each hexapole applies a three-fold magnetic field with a different azimuth, whereby the entire system corrects spherical and geometrical aberrations such as threefold astigmatism at the same time ${ }^{[13]}$.

This correction system has largely contributed to the improved spatial resolution of conventional TEM and STEM that are generally designed for operation at $100 \mathrm{kV}$ or higher.
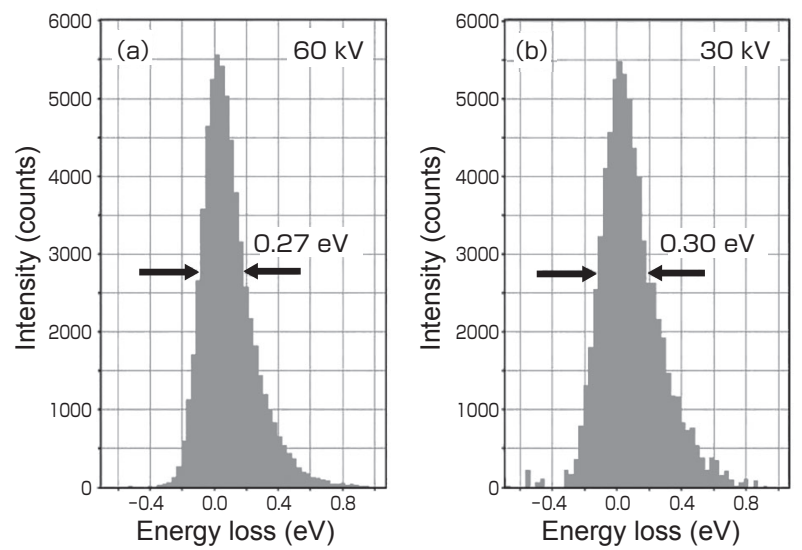

Fig. 3 Evaluation of energy spread of low-voltage electron gun

Recorded at acceleration voltages of (a) $60 \mathrm{kV}$ and (b) $30 \mathrm{kV}$ 
However, assuming that the application of this corrector to our microscope at $30-60 \mathrm{kV}$ in Triple-C project, it was predicted that the spatial resolution would be limited by the residual six-fold astigmatism, which is fundamentally inevitable in the case of this double-hexapole system. Therefore, we started to search for a completely new method to enable the correction of both spherical aberration and high-order geometrical astigmatisms up to six-fold, and finally developed an innovative correction system consisting of triple magnetic dodecapoles and transfer lenses ${ }^{[15]-[17]}$.

Figure 4 shows the construction and calculated electronbeam trajectories of the conventional double-hexapole type and our new triple-dodecapole type (named as Delta type) correctors $^{[17]}$. For the calculated trajectory of the conventional corrector (a), six-fold symmetry is more obvious in the higher angle region at the end of the second hexapole. This is caused by the six-fold astigmatism that is inevitably generated by the combination of three-fold magnetic fields between the double hexapoles as described above. Such six-fold astigmatism is also generated between the first and second dodecapoles of the Delta corrector, and between the second and third dodecapoles as well. In this case, however, the astigmatisms can be completely canceled out by carefully adjusting the azimuth of the magnetic field in each dodecapole. As shown in Fig. 4 (b), the calculated trajectory for the Delta corrector (b) is almost circular even at higher angles at the end of the third dodecapole, indicating that the residual six-fold astigmatism is negligible.

(a) Double-hexapole type

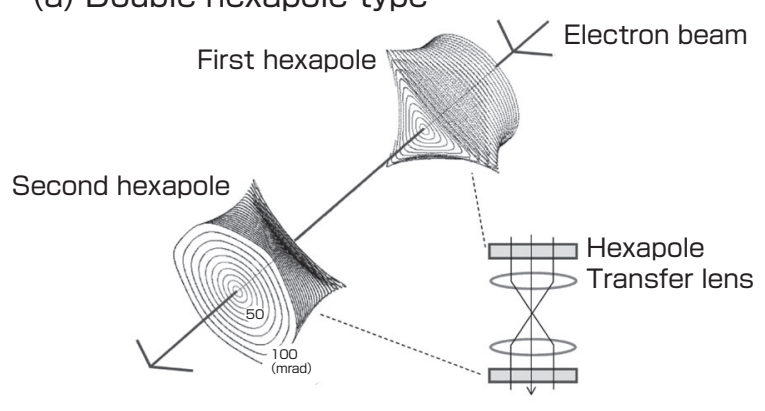

(b) Triple-dodecapole "Delta" type

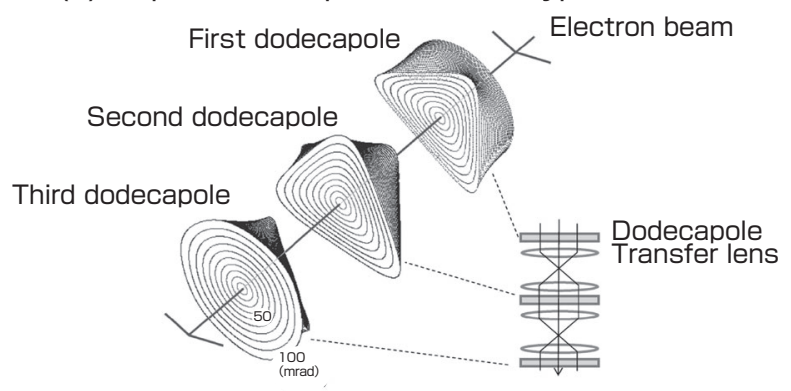

Fig. 4 Construction and calculated electron-beam trajectories of spherical aberration correctors

(a) Double-hexapole type, (b) Delta (triple-dodecapole) type

\subsection{Chromatic aberration corrector}

Through the Triple-C project, we have developed a new chromatic aberration corrector for TEM in addition to a Delta spherical aberration corrector. As described in subchapter 3.1, the degree of electron-beam broadening due to chromatic aberration is proportional to $\Delta E / E$, which implies that spatial resolution is more seriously affected at a lower $E$. Therefore, chromatic aberration correction of the objective lens significantly contributes toward improving spatial resolution and image quality at $30 \mathrm{kV}$, even when the microscope is equipped with our new low-voltage CFEG. In this project, we developed a completely new system for chromatic aberration correction, for which two thick quadrupole fields are applied to generate a combination concave-lens effect ${ }^{[17]-[19]}$.

Figure 5 shows the calculated electron-beam trajectory of our new chromatic aberration corrector consisting of two thick dodecapoles and several transfer lenses. Each dodecapole of this corrector is used to superimpose magnetic and electrostatic quadrupole fields, where the deflection sensitivity of an electron beam depends on the acceleration voltage in a different manner compared to the magnetic field of the objective lens. The two-fold stigmatism introduced at the first dodecapole is canceled at the second dodecapole, and the entire system functions as a concave lens with negative chromatic aberration. We installed this corrector in the microscope and examined its performance by slightly changing the acceleration voltage from $30 \mathrm{kV}$. We found that the defocus remained unchanged even when the acceleration voltage was changed by $\pm 0.025 \mathrm{kV}$, which confirms that the chromatic aberration was successfully corrected.

\section{Integration of elemental technologies}

\subsection{Construction of low-voltage electron microscopes}

We constructed experimental systems of low-voltage electron microscopes by integrating the elemental technologies described above and then examined their performances. Two microscopes with different constructions optimized for their use were developed separately, enabling us to efficiently proceed with their examination and reach the stage of

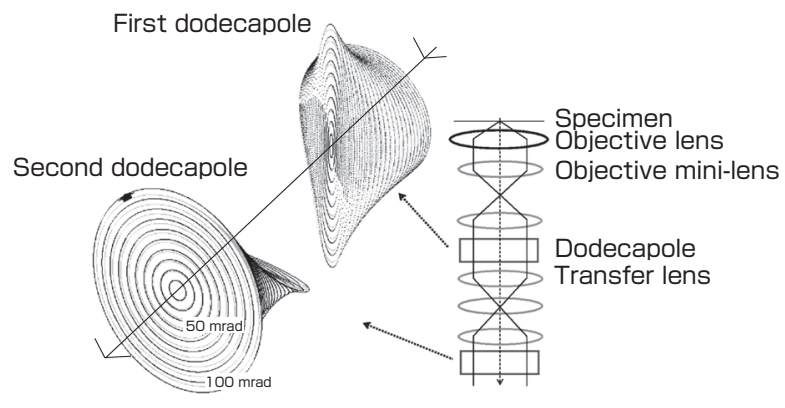

Fig. 5 Construction and calculated electron-beam trajectory of new chromatic aberration corrector 
their practical application as early as possible. The major components of these experimental microscopes are listed in Table 1. The first microscope is a spherical aberrationcorrected TEM/STEM for 30-60 kV, while the second microscope is a spherical and chromatic aberration-corrected TEM for $30 \mathrm{kV}$. They were initially developed as specially designed low-voltages microscopes. We obtained valuable results regarding their performances as detailed below.

\subsection{Performance of the first low-voltage microscope (TEM/STEM)}

We started the operation of our first experimental microscope (Fig. 6) equipped with two Delta spherical aberration correctors for TEM and STEM modes in 2008. Through our preliminary experiments using a conventional electron gun (Schottky-type FEG) for a $200 \mathrm{kV}$-class commercial microscope, we confirmed that spherical aberration and high-order geometrical astigmatisms up to six-fold were canceled by the Delta correctors both in TEM and STEM modes. We then installed the new low-voltage CFEG and an EELS spectrometer in the microscope.

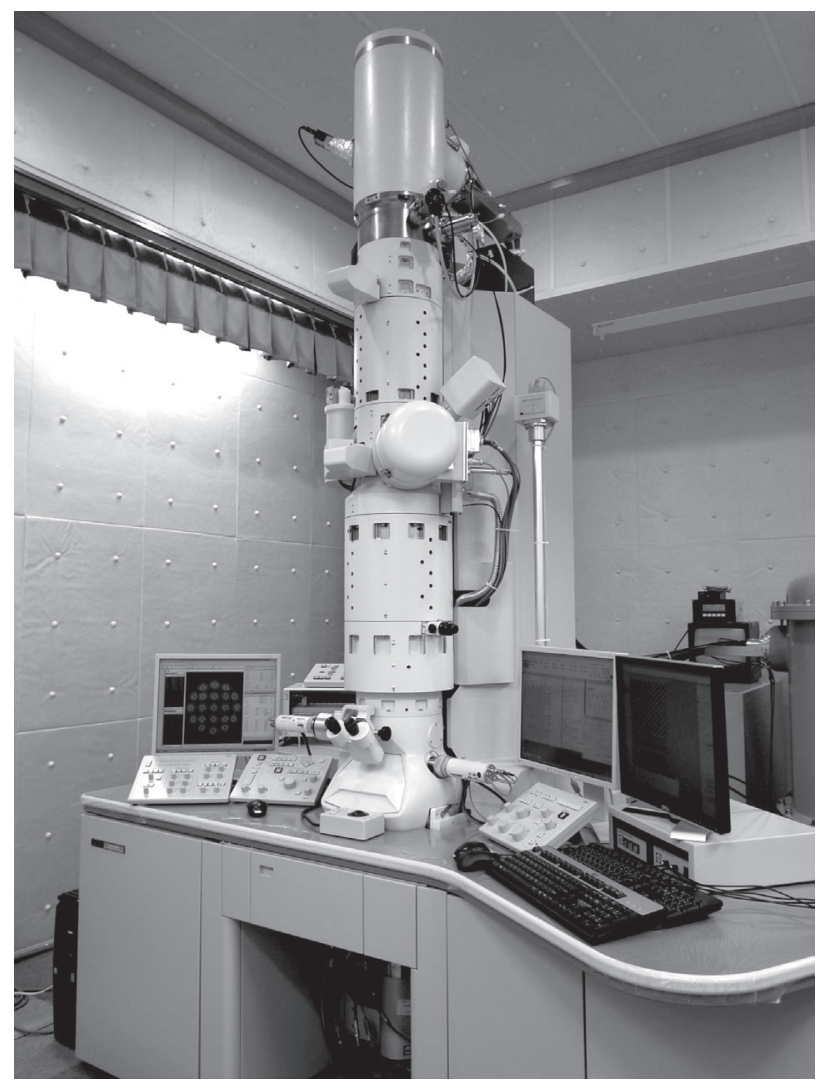

Fig. 6 External view of the first experimental microscope (spherical aberration-corrected lowvoltage TEM/STEM)
Table 1. Main functions and constitution of experimental low-voltage electron microscopes

\begin{tabular}{lcc}
\hline & First system & Second system \\
\hline Main function(s) & TEM/STEM/EELS & TEM \\
Acceleration voltage(s) (kV) & 60,30 & 30 \\
Low-voltage electron gun & 0 & 0 \\
Spherical aberration corrector (for STEM) & 0 & - \\
Spherical aberration corrector (for TEM) & 0 & 0 \\
Chromatic aberration corrector (for TEM) & - & 0 \\
Spectrometer for EELS & 0 & - \\
\hline : equipped, - : not equipped & &
\end{tabular}

We evaluated the spatial resolution of this microscope in STEM mode by using it to observe silicon $(\mathrm{Si})<110>$, which is conventionally used as a standard sample for high-resolution $\mathrm{STEM}^{[11]}$. Both of the annular dark field (ADF-) STEM images observed at 60 and $30 \mathrm{kV}$ (Fig. 7) clearly show the so-called dumbbell structures, where the projected atomic columns in each "dumbbell" are separated by $136 \mathrm{pm}$. Furthermore, the smallest lattice spacing observed here was 96 and $111 \mathrm{pm}$ at 60 and 30 $\mathrm{kV}$, respectively, as shown by fast Fourier transformation (FFT) of the ADF images. Regarding these distances as spatial resolution $d$, the ratios of $d$ to the wavelengths of electrons $\lambda, d / \lambda$, were found to be 20 and 17 at 60 and $30 \mathrm{kV}$, respectively. Our microscope thus exhibited the highest spatial resolution in terms of $d / \lambda$, which exceeded the past record of $d / \lambda=25$, corresponding to $d=0.05 \mathrm{~nm}$ obtained at $300 \mathrm{kV}^{[10]}$.

We then examined spatial resolution of the microscope in TEM mode by using it to observe gold $(\mathrm{Au})$ nanoparticles ${ }^{[11]}$. Lattice fringes of $\mathrm{Au}<200>$ with a separation of $204 \mathrm{pm}$ were unambiguously imaged both at 60 and $30 \mathrm{kV}$ (Fig. 8), and smaller lattice spacing of 79 and $91 \mathrm{pm}$ were also resolved at 60 and $30 \mathrm{kV}$, respectively, as shown by FFT of the TEM
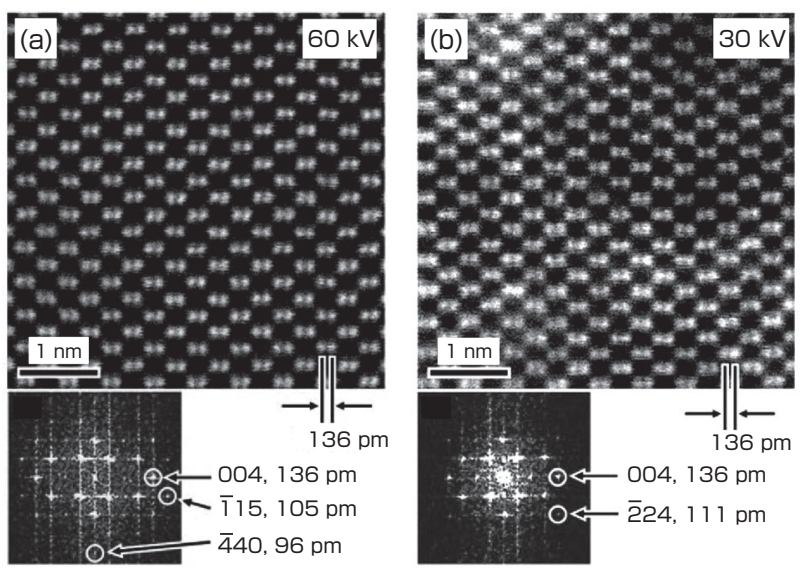

Fig. 7 Performance examination of the first low-voltage microscope in STEM mode

Recorded at acceleration voltages of (a) $60 \mathrm{kV}$ and (b) $30 \mathrm{kV}$ $\mathrm{Si}<110>$ was used as test specimen. 
images. These results show the excellent spatial resolution of our microscope in TEM mode as well as in STEM mode. Comparing the TEM image of a SWCNT obtained at $30 \mathrm{kV}$ (Fig. 9) with those of conventional microscopes (e.g. Fig. 1), we can easily recognize that the performance of our microscope is comparable to existing spherical aberration-corrected TEM systems operated at $120 \mathrm{kV}$.

\subsection{Performance of the second low-voltage microscope (TEM)}

Our second experimental microscope is a $30 \mathrm{kV}$-TEM equipped with a chromatic aberration corrector in tandem with a Delta spherical aberration corrector. As described in subchapter 3.3, its chromatic aberration corrector has an innovative optical system based on the combination concavelens effect of superposed electrostatic and magnetic fields. We checked the fundamental operation of this microscope
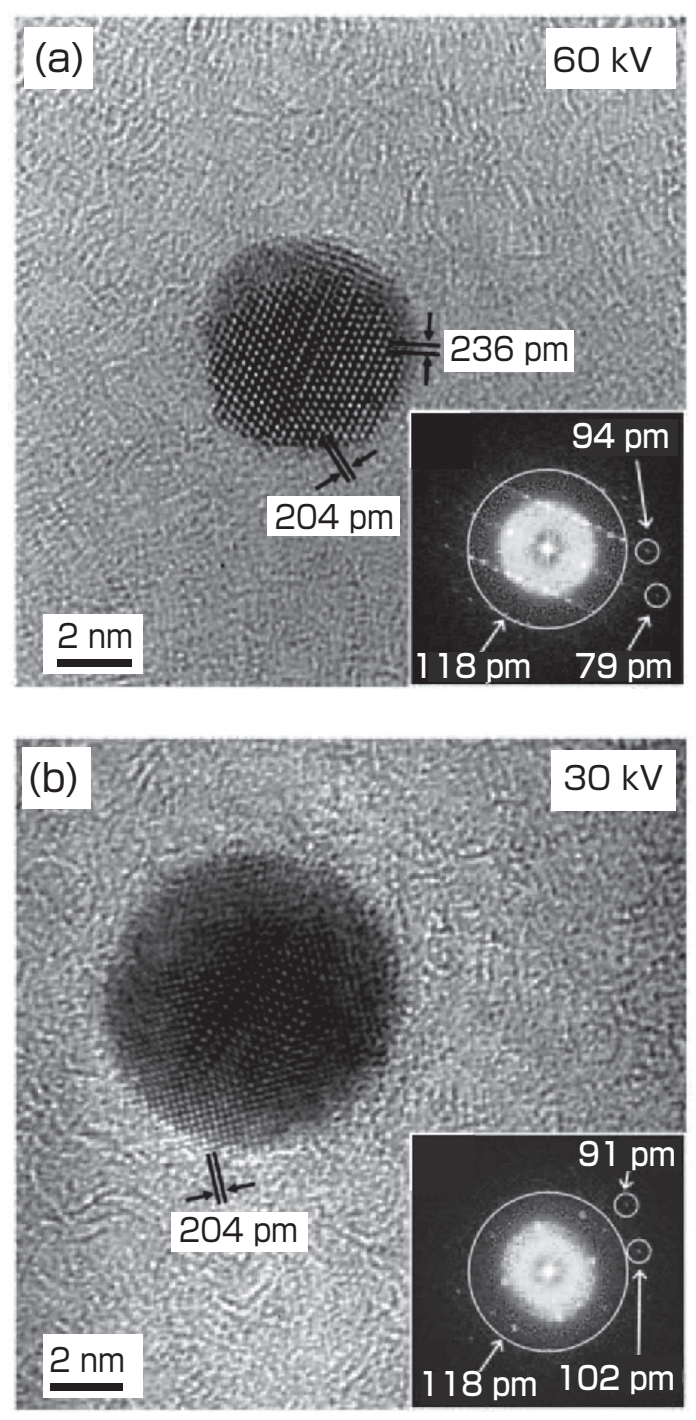

Fig. 8 Performance examination of the first low-voltage microscope in TEM mode

Recorded at acceleration voltages of (a) $60 \mathrm{kV}$ and (b) $30 \mathrm{kV}$ Gold nanoparticles were used as test specimens. such as aberration correction in 2010, taking measures to reduce electric noise to improve its stability. We are currently verifying the effect of simultaneously correcting chromatic and spherical aberrations in TEM observation for various standard samples.

Figure 10 shows a TEM image of $\mathrm{Si}<110>$ recorded by using this microscope temporarily equipped with a conventional electron gun. Even small lattice spacing of $125 \mathrm{pm}$ was resolved, as shown by FFT, suggesting that the spatial resolution of this microscope is already comparable with that of our first microscope operated in TEM mode at the same voltage of $30 \mathrm{kV}$. We expect that the performance of this second microscope will be further improved to achieve higher resolutions by installing a new low-voltage CFEG (completed in September 2011) and by optimizing the alignment of the optical system including two aberration correctors.

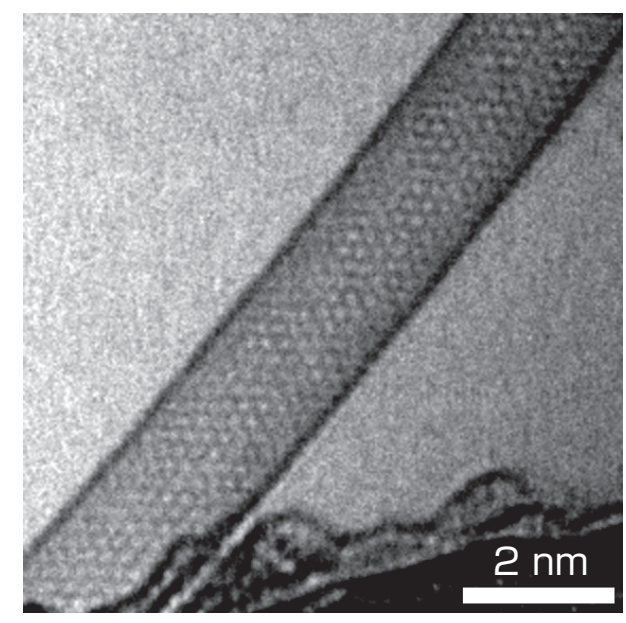

Fig. 9 TEM image of a SWCNT recorded at an acceleration voltage of $30 \mathrm{kV}$

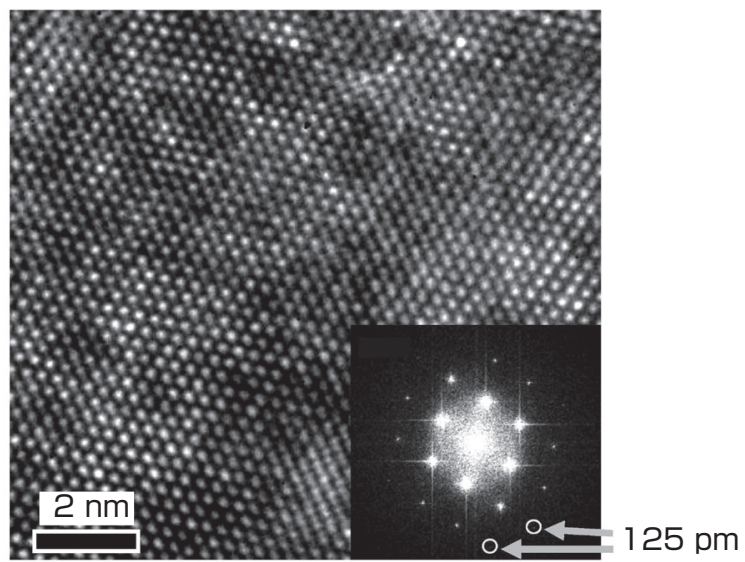

Fig. 10 Performance examination of the second lowvoltage microscope (TEM)

Recorded at an acceleration voltage of $30 \mathrm{kV}$

$\mathrm{Si}<110>$ was used as test specimen. 


\section{Application of low-voltage microscopes and their problems}

We have already completed the fundamental performance examination of our first microscope, which was developed prior to the second microscope, and have started using it to make scientific observations. In this chapter, we describe some typical results obtained by using the first microscope along with the problems associated with its application.

\subsection{Identifying single metal atoms inside fullerenes}

We carried out the STEM-EELS analysis of fullereneincorporated SWCNTs (so-called nano-peapods) using our first microscope to detect individual metal ions encapsulated inside the fullerene cages and to identify their elements. Due to the serious irradiation-induced damage of fullerenes, which causes rapid coalescence and/or opening of their carbon cages $^{[20][21]}$, it was impossible to directly observe the original isolated state of these metal ions in previous STEM observations made at $100 \mathrm{kV}$ or higher ${ }^{[22]}$. Our microscope enables the STEM-EELS analysis of nano-peapods with drastically reduced damage at $60 \mathrm{kV}^{[23]}$.

Figure 11 shows the results of the STEM-EELS analysis for a nano-peapod sample containing calcium-encapsulated fullerenes $\mathrm{Ca} @ \mathrm{C}_{82}$. In the bright-field (BF) STEM image (a), the structures of seven fullerene molecules appear to be maintained without undergoing significant beam damage. Seven calcium ions $\left(\mathrm{Ca}^{2+}\right)$ inside these carbon cages are not clearly seen in this image due to their low contrast, but they are successfully detected by EELS elemental mapping for calcium (b), as indicated by the arrows. We thus found that low-voltage STEM-EELS enables the direct detection and elemental identification of individual $\mathrm{Ca}^{2+}$ ions with drastically reduced beam damage to the samples. Such a technique will greatly contribute to the analysis of biological specimens, especially in the elucidation of the mechanisms of ion channels, which play important roles in (a)

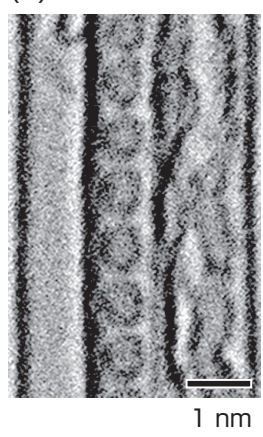

(b)

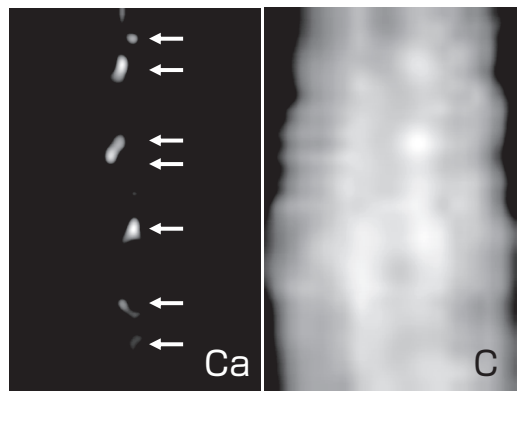

Fig. 11 Elemental analysis with low-voltage STEM-EELS (a) ADF-STEM image, (b) elemental maps for calcium and carbon (left and right, respectively)

Nano-peapods of calcium-encapsulated fullerenes $\mathrm{Ca} @ \mathrm{C}_{82}$ were analyzed at $60 \mathrm{kV}$. neurotransmission. Although the observation of ion channels has often been attempted by using electron microscopes in the past, identifying individual ions inside and obtaining high-resolution images of the channel structures has never been achieved because of the serious beam damage that occurs at higher acceleration voltages. The high-performance, low-voltage electron microscopy that we detailed here should be indispensable for the atomic-level characterization of structures and mechanisms inherent in biological specimens.

\subsection{Mapping the electronic state on a graphene edge} Graphene is a single layer of carbon atoms that forms a honeycomb network. Owing to its unique and excellent properties such as its theoretically predicted and/or experimentally proven electronic characteristics, graphene is now regarded as a strong candidate for functional materials to be used in constructing next-generation nano-electronics. Because electronic properties of graphene significantly depend on the atom arrangement on its edge, it is quite important to understand precisely the correlation between local structures and electronic states in this material. Through the Triple-C project, we succeeded in STEM-EELS mapping of the electronic states of graphene edges without introducing serious beam damage ${ }^{[24]}$.

Figure 12 shows the results of STEM-EELS analysis for a peripheral area of graphene at $60 \mathrm{kV}$. Possible local structures of three carbon atoms indicated by the colored arrows in the ADF-STEM image (a) are illustrated in (b), and the EELS spectra observed at these atoms are shown in (c). Note that two carbon atoms on the edge (colored blue and red) give characteristic EELS peaks at different energies (indicated by the black arrows), which were not observed for an inner carbon atom (colored green). We ascribed these EELS peaks to the electronic states peculiar to the carbon atoms on the graphene edge. Our atomic-level analysis thus provided the first direct proof that carbon atoms on a graphene edge possess different electronic states from those of inner atoms on the same layer.

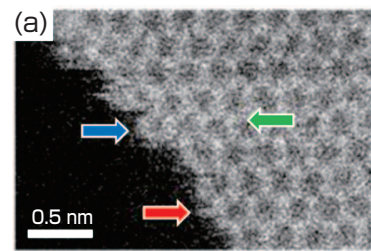

(b)

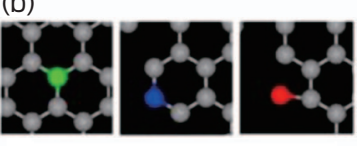

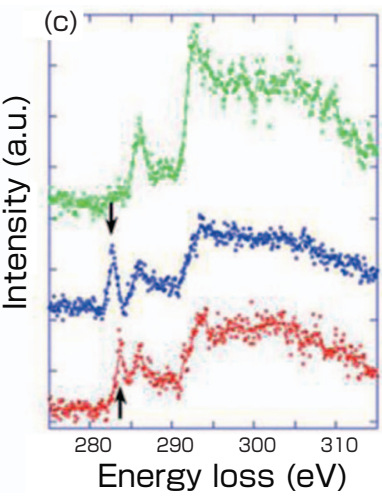

Energy loss (eV)
Fig. 12 Observation of electronic states with lowvoltage STEM-EELS

(a) ADF-STEM image, (b) possible local structures, (c) EELS spectra of individual carbon atoms

Graphene was analyzed at $60 \mathrm{kV}$. 


\subsection{Known practical problems with low-voltage microscopy}

In the experiments described above, we found some practical problems associated with our low-voltage microscope that would not occur for conventional observations made at higher acceleration voltages without aberration correction. For example, the etching effect of residual gaseous species in the microscope column, which causes the gradual loss of atoms in a sample during observation, is more obvious at lower voltages, while the knock-on effect of electrons itself is drastically reduced. In addition, even a slight change in condition of the CFEG emitter can significantly affect the spatial resolution and image quality of the microscope, where spherical aberration and various astigmatisms are finely corrected by the Delta corrector. We are currently taking measures to solve these problems by reinforcing the vacuum system of the microscope, and by optimizing the emitter shape and its operating conditions. Since we have proven the potential of our low-voltage microscopes as described above, our final goal is to improve them to the level that they can stably and easily exhibit their highest performance even for practical uses such as for material research and development.

\section{Future prospects}

In this paper, we reviewed our Triple-C project, through which we have developed completely new low-voltage electron microscopes for the first time. The advantage of using low acceleration voltages was not recognized in the field of electron microscopy when we started the concrete planning of this project in 2006. However, the situation completely changed within a few years of launching this project. A foreign group has also started developing lowvoltage microscopes based on a similar concept ${ }^{[25]}$, and some existing projects originally planned to improve the performance of middle-voltage microscopes for $80-300 \mathrm{kV}$ have been modified for low-voltage systems ${ }^{[26][27]}$. Low-voltage TEM and STEM are now regarded as the main advanced forms of electron microscopy, and the competition between full-scale projects to develop such microscopes is intensifying worldwide. Each project is aimed at achieving a spatial resolution close to 0.1 $\mathrm{nm}$ at $50 \mathrm{kV}$ or lower by using newly developed chromatic aberration correctors such as those employed in our project or by introducing conventional monochrometers. Low-voltage microscopes can be considered an innovation in electron microscopy that follows spherical aberration correctors developed in 1990s. These innovations are expected to satisfy the growing requirement for microscopes to be able to observe new targets such as nano-materials and single organic molecules.

As low-voltage microscopes become widespread and are put into practical use, they are expected to contribute significantly in the characterization of various kinds of materials, especially the materials used in the fields of chemistry and biology. If these microscopes can enable us to more easily observe the dynamic behavior of molecules and atoms, we can immediately apply them to important investigations, such as the structure analysis of ion channels and the in-situ observation of catalytic reactions. For example, direct observation of the reconstruction of individual molecules in the presence of metal clusters should help us further understand the mechanisms responsible for catalytic reactions at an atomic level, thereby making a huge impact on society. If we can observe in real time how a particular functional group in a molecule is excited by light or heat and how the electronic state of a particular atom is changed, we will be able to obtain fundamental data through which we can elucidate the mechanisms responsible for various chemical reactions at the atomic level.

Low-voltage electron microscopy and spectroscopy, which cause less irradiation damage to samples, are beneficial for analyzing not only soft matter but also inorganic materials. When characterizing crystalline materials, for example, they can provide us with new viewpoints on the physical properties of samples such as the formation and annihilation processes associated with point defects. We may also be able to gain a better understanding of the correlation between the atomic-level structures and the electronic states of individual quantum objects such as CNTs and fullerenes based on high-precision EELS analyses.

\section{Acknowledgements}

We thank Koji Kimoto (NIMS) for his cooperation throughout the Triple-C project; Eiji Okunishi (JEOL) for his cooperation during the low-voltage EELS experiments; and Hiromichi Kataura, Toshiya Okazaki, Yoko Iizumi, and Haruka Kobayashi (AIST) for their help with sample preparation. A portion of the experiments performed with the low-voltage microscope was financially supported by KAKENHI (19054017 and 23750250).

\section{References}

[1] S. Horiguchi: Ko-bunkaino Denshi Kenbikyo (HighResolution Electron Microscopy), Kyoritsu Shuppan, Tokyo (1988) (in Japanese).

[2] D. B. Williams and C. B. Carter: Transmission Electron Microscopy (2nd Ed.), Springer (2009).

[3] A. Hashimoto, H. Yorimitsu, K. Ajima, K. Suenaga, H. Isobe, J. Miyawaki, M. Yudasaka, S. Iijima and E. Nakamura: Selective deposition of a gadolinium (III) cluster in a hole opening of single-wall carbon nanohorn, Proc. Natl. Acad. Sci. USA, 101, 8527-8530 (2004).

[4] A. Hashimoto, K. Suenaga, A. Gloter, K. Urita and S. Iijima: Direct evidence for atomic defects in grapheme layers, Nature, 430, 870-873 (2004).

[5] K. Suenaga, H. Wakabayashi, M. Koshino, Y. Sato, K. Urita and S. Iijima: Imaging active topological defects in carbon nanotubes, Nat. Nanotechnol., 2, 358-360 (2007). 
[6] Y. Sato, K. Suenaga, S. Okubo, T. Okazaki and S. Iijima: Structures of $D_{5 d^{-}}-\mathrm{C}_{80}$ and $I_{\mathrm{h}}-\mathrm{Er}_{3} \mathrm{~N} @ \mathrm{C}_{80}$ fullerenes and their rotation inside carbon nanotubes demonstrated by aberration-corrected electron microscopy, Nano Lett., 7, 3704-3708 (2007).

[7] Y. Sato, K. Yanagi, Y. Miyata, K. Suenaga, H. Kataura and S. Iijima: Chiral-angle distribution for separated single-walled carbon nanotubes, Nano Lett., 8, 3151-3154 (2008).

[8] http://www.busshitu.jst.go.jp/kadai/year03/team03.html

[9] B. Kabius, P. Hartel, M. Haider, H. Müller, S. Uhlemann, U. Loebau, J. Zach and H. Rose: First application of Cccorrected imaging for high-resolution and energy-filtered TEM, J. Electron Microsc., 58, 147-155 (2009).

[10] H. Sawada, Y. Tanishiro, N. Ohashi, T. Tomita, F. Hosokawa, T. Kaneyama, Y. Kondo and K. Takayanagi: STEM imaging of 47-pm-separated atomic columns by a spherical aberrationcorrected electron microscope with a $300-\mathrm{kV}$ cold field emission gun, J. Electron Microsc., 58, 357-361 (2009).

[11] T. Sasaki, H. Sawada, F. Hosokawa, Y. Kohno, T. Tomita, T. Kaneyama, Y. Kondo, K. Kimoto, Y. Sato and K. Suenaga: Performance of low-voltage STEM/TEM with delta corrector and cold field emission gun, J. Electron Microsc., 59, S7-S13 (2010).

[12] H. Rose: Outline of a spherically corrected semiaplanatic medium-voltage transmission electron-microscope, Optik, 85, 19-24 (1990).

[13] M. Haider, S. Uhlemann, E. Schwan, H. Rose, B. Kabius and K. Urban: Electron microscopy image enhanced, Nature, 392, 768-769 (1998).

[14] F. Hosokawa, T. Sannomiya, H. Sawada, T. Kaneyama, Y. Kondo, M. Hori, S. Yuasa, M. Kawazoe, Y. Nakamichi, T. Tanishiro, N. Yamamoto and K. Takayanagi: Design and development of Cs corrector for a $300 \mathrm{kV}$ TEM and STEM, Proc. IMC 16 (Sapporo), 582 (2006).

[15] H. Sawada, T. Sasaki, F. Hosokawa, S. Yuasa, M. Terao, M. Kawazoe, T. Nakamichi, T. Kaneyama, T. Tomita, Y. Kondo, K. Kimoto and K. Suenaga: Correction of higher order geometrical aberration by triple 3 -fold astigmatism field, $J$. Electron. Microsc., 58, 341-347 (2009).

[16] H. Sawada, T. Sasaki, F. Hosokawa, S. Yuasa, M. Terao, M. Kawazoe, T. Nakamichi, T. Kaneyama, Y. Kondo, K. Kimoto and K. Suenaga: Higher-order aberration corrector for an image-forming system in a transmission electron microscope, Ultramicroscopy, 110, 958-961 (2010).

[17] H. Sawada, F. Hosokawa, T. Sasaki, T. Kaneyama, Y. Kondo and K. Suenaga: Aberration correctors developed under the Triple C project, in P. Hawkes (Ed.): Advances in Imaging and Electron Physics, 168, 297-336 (2011).

[18] F. Hosokawa, H. Sawada, T. Sasaki, Y. Kondo and K. Suenaga: Chromatic aberration correction of objective lens using a concave lens effect of a long quadrupole field, Proceedings of the 66th annual meeting of the Japanese Society of Microscopy (Nagoya), 14 (2010) (in Japanese).

[19] H. Sawada, F. Hosokawa, T. Sasaki, S. Yuasa, M. Kawazoe, M. Terao, T. Kaneyama, Y. Kondo, K. Kimoto and K. Suenaga: Chromatic aberration correction by combination concave lens, Microsc. Microanal., 16(S2), 116-117 (2010).

[20] K. Urita, Y. Sato, K. Suenaga, A. Gloter, A. Hashimoto, M. Ishida, T. Shimada, H. Shinohara and S. Iijima: Defect-induced atomic migration in carbon nanopeapod: Tracking the singleatom dynamic behavior, Nano Lett., 4, 2451-2454 (2004).

[21] Y. Sato, T. Yumura, K. Suenaga, K. Urita, H. Kataura, T. Kodama, H. Shinohara and S. Iijima: Correlation between atomic rearrangement on defective fullerenes and migration behaviors of encaged metal ions, Phys. Rev. B, 73, 233409 (4 pages) (2006).
[22] K. Suenaga, M. Tencé, C. Mory, C. Colliex, H. Kato, T. Okazaki, H. Shinohara, K. Hirahara, S. Bandow and S. Iijima: Element-selective single atom imaging, Science, 290, 2280-2282 (2000).

[23] K. Suenaga, Y. Sato, Z. Liu, H. Kataura, T. Okazaki, K. Kimoto, H. Sawada, T. Sasaki, K. Omoto, T. Tomita, T. Kaneyama and Y. Kondo: Visualizing and identifying single atoms using electron energy-loss spectroscopy with low accelerating voltage, Nat. Chem., 1, 415-418 (2009).

[24] K. Suenaga and M. Koshino: Atom-by-atom spectroscopy at graphene edge, Nature, 468, 1088-1090 (2010).

[25] http://www.salve-project.de/

[26] http://ncem.lbl.gov/TEAM-project/

[27] http://www.superstem.org/

\section{Authors}

\section{Yuta SATO}

Recieved his Ph.D. in energy science from Kyoto University in 2004. He joined AIST in 2004, and is currently a researcher of Nanotube Research Center, AIST. In this project, he is mainly in charge of application experiments of the low-voltage electron microscope.

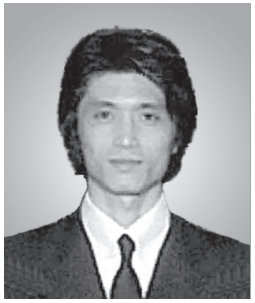

\section{Takeo SASAKI}

Received his Ph.D. in materials engineering from the University of Tokyo in 2006. He joined JEOL Ltd. in 2006, and is currently a chief of EM Technical Group 1, EM Business Unit, JEOL. In this project, he is mainly in charge of performance examination and application experiments of the low-voltage electron microscopes.

\section{Hidetaka SAWADA}

Received his Ph.D. in materials engineering from the University of Tokyo in 2002. He joined JEOL Ltd. in 2002, and is currently a researcher of EM Technical Group 1, EM Business Unit, JEOL. In this project, he is mainly in charge of development and performance examination of the low-voltage electron microscopes.

\section{Fumio HosoKawa}

Recieved his B.S. from Kyushu University in 1984. He joined JEOL Ltd. in 1984, and is currently a senior researcher of EM Technical Group 1, EM Business Unit, JEOL. His primary duty is to develop optical systems of transmission electron microscopes, especially aberration correctors. In this project, he is mainly in charge of designing the chromatic aberration corrector.
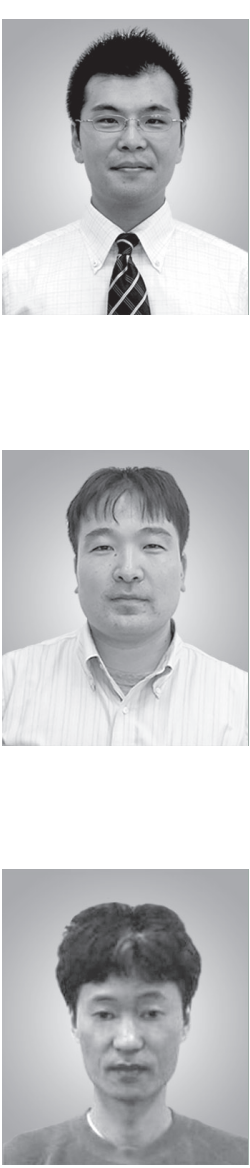
Takeshi ToMITA

Recieved his B.S. from Kyushu University in 1972. He joined JEOL Ltd. in 1972, and is currently a member of EM Technical Group 1, EM Business Unit, JEOL. In this project, he is mainly in charge of development of the low-voltage field emission guns.

\section{Toshikatsu Kaneyama}

Received his M.S. from Tohoku University in 1987. He joined JEOL Ltd. in 1987, and is currently the general manager of EM Technical Group 1, EM Business Unit, JEOL. In this project, he is responsible for management of developing the lowvoltage electron microscopes.

\section{Yukihito Kondo}

Received his M.E. from Tokyo Institute of Technology in 1978. He joined JEOL Ltd. in 1979, and is currently the technical general manager of EM Business Unit. In this project, he is responsible for general management of developing the lowvoltage electron microscopes.

\section{Kazutomo Suenaga}

Received his Ph.D. in materials science from the University of Tokyo in 1994. He was a postdoctoral fellow at Ecole Nationale Superieure des Mine de Paris, France (1994-1997), and at the Solid State Physics Laboratory in the University Paris-Sud, France (1997-1998). Then he joined the Japan Science and Technology Corporation (JST) as a researcher (1998-
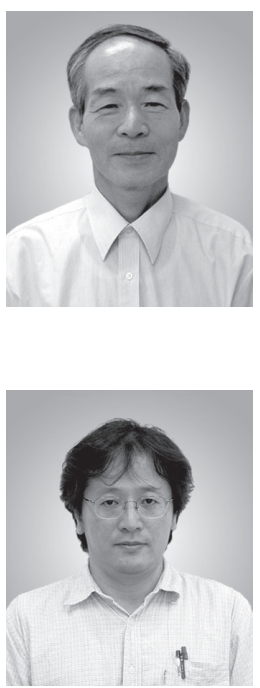

2001). He has been a team leader at Nanotube Research Center of AIST since 2001, and also a prime senior researcher at AIST since 2010. He is responsible for administration of this project, and also in charge of application experiments of the low-voltage electron microscopes.

\section{Discussions with Reviewers}

\section{Author contributions}

Question (Shuji Abe, Evaluation Department, AIST; Toshimi Shimizu, Nanotechnology, Material and Manufacturing, AIST)

This paper is co-written by eight authors, and mainly presents the results of the collaboration between AIST and JEOL. We understand that this work has been carried out as a part of the JST-CREST joint project in which AIST, JEOL and NIMS are participating. We expect author profiles are provided above, but can you explain the contributions of these organizations and members?

Answer (Yuta Sato)

This project has been carried out through collaboration between JEOL, NIMS and AIST. Eight researchers from each of JEOL and AIST, and two from NIMS have participated in this project (in total, excluding technical assistant staffs). In order to realize unprecedented low-voltage electron microscopes within a given period, we have had to combine our expertise in the fields such as theory of electron microscopy and spectroscopy, equipment, materials and phenomena to observe (solid-state physics, material science, nano-science, biology, etc.). The JEOL team, as a microscope manufacturer, developed each component, constructed two microscopes and examined their performances. The NIMS and AIST teams took charge of preliminary study for planning this project, the application of the low-voltage microscopes to scientific observation, and reference experiments using conventional microscopes. We described these contributions in Chapter 2 of the revised manuscript.

\section{Competitors' projects Question (Toshimi Shimizu)}

This paper shows that the authors' group has pioneered in developing low-voltage electron microscopes equipped with chromatic and/or spherical aberration correctors. In chapter 6, however, it is also stated that low-voltage microscopes are now regarded as the main advanced forms of electron microscopy, and the competition between full-scale projects is intensifying worldwide. It is not clear to me whether such a world trend originated from the authors' project, or whether it independently emerged as integration of newly developed elemental technologies. I think additional description on the world trend and on the benchmarks of this project is effective in presenting more clearly the features of this project.

\section{Answer (Kazutomo Suenaga)}

In our revised manuscript, we emphasized that this is the first project in the world to construct totally new low-voltage electron microscopes. In addition, we stated that a foreign group also started developing low-voltage microscopes based on a similar concept just after we launched this project, and some other existing projects decided to cover low-voltage systems as additional targets. We presume that the performance of our first experimental microscope, which is the world's first system specially designed for low acceleration voltages, has affected the competitors' projects both directly and indirectly. Although it is difficult at this stage to specify how far such effects have spread over, we revised chapter 6 to refer to the world trend according to your comment.

\section{3 "Soft matter"}

\section{Question (Shuji Abe)}

I find that the term "soft matter" often appears in this paper, while the application of the low-voltage microscopes is currently focused on carbon nano-materials. In my opinion, carbon nanomaterials themselves are not classified as soft matter, because they are known as robust materials. For future application of your lowvoltage microscopes to real soft matter such as bio-materials, do you think their performance is high enough even at this stage, or further technical improvement is necessary?

\section{Answer (Yuta Sato)}

Low-voltage observation of soft matter is a final goal of our project. In our preliminary experiments, we used carbon nano-materials as test specimens to examine the effect of low acceleration voltages. CNTs and graphene are known to possess high mechanical strengths, and expected to be more stable under electron-beam irradiation than soft matters in general. Nevertheless, we had to give up many scientifically important experiments on these materials in the past, due to serious irradiation-induced damages occurring in conventional microscopes even at relatively low voltages such as 120 and $80 \mathrm{kV}$. In this project, we have achieved drastic reduction 
of such damages and improvement of sensitivity by newly developing low-voltage microscopes, which enable EELS analysis of single carbon atoms, for example. Performance of our low-voltage microscopes in respect of spatial resolution has already been improved to a high level as we originally aimed. Our project now enters the next stage, where the microscopes are applied to observation of soft matter to further examine the effect of reducing beam damages. At this stage, it is quite important to elucidate irradiation-induced damages on soft matter and their unknown mechanisms based on the observed data, and to optimize operation condition of the microscopes such as acceleration voltage and beam current. This final stage is essential to promote practical use of low-voltage microscopes, and we recognize that it is our duty, especially of AIST team, to contribute from both standpoints of developers and users.

\section{Practical use of low-voltage microscopes}

Question (Toshimi Shimizu)

Once the observation of various soft matters with high sensitivity is realized by using low-voltage electron microscopes, it should have not only a great impact on the researchers in this field but also considerable ramifications on other fields such as material and life sciences. From the results presented here, it seems that the first low-voltage microscope of this project can immediately be applied to practical use even at the present state. I think the status of this project as Full Research would be more clearly shown, if you mention the processes to realize practical use of the microscopes and to solve the existing problems.

Answer (Yuta Sato)

Our first microscope has stably exhibited excellent performance at the present stage of the project, convincing us that its practical use will be realized in the near future. At the same time, we recognize that some factors of the microscope can affect its performance more seriously than expected by disturbing the observation or limiting the spatial resolution. It is essential to solve such problems for future application of the microscope, so we have inserted subchapter 5.3 into the revised manuscript to state the current problems and measures we are taking to solve them. 